\title{
Microstructure Analyses of Metal-Filled Carbon Nanotubes Synthesized by Microwave Plasma-Enhanced Chemical Vapor Deposition
}

\author{
Yasuhiko Hayashi, Tomoharu Tokunaga, Kenji Kaneko, Simon J. Henley, Vlad Stolojan, J. David Carey, and \\ S. R. P. Silva
}

\begin{abstract}
Pd/Co-based metal-filled carbon nanotubes (MF-CNTs) were synthesized by a microwave plasma-enhanced chemical vapor deposition method using a bias-enhanced growth technique. Pd/Co-based MF-CNTs were analyzed by scanning electron microscopy (SEM), transmission electron microscopy (TEM) electron energy loss spectroscopy (EELS), and Raman spectroscopy. MF-CNTs were well-aligned and uniform in size on a Si substrate. Both multiwall nanotube carbon nanotubes (CNTs) and herringbone (or stacked cups structure) structures were observed. High-resolution TEM revealed that MF-CNTs were composed of highly ordered graphite layers, and the elemental maps of EELS indicate that both Co and Pd metals are present inside the nanotubes. TEM results clearly showed that both Pd and Co metals were successfully encapsulated into the CNTs. We observed a low value for the Raman intensity ratio between $D\left(1355 \mathrm{~cm}^{-1}\right)$ and $G\left(1590 \mathrm{~cm}^{-1}\right)$ bands with no shift of the G-peak position and no broadening of the G-peak, indicative of high-quality Pd/Co-based MF-CNTs. Based on TEM characterization, we propose a description for the encapsulating mechanisms.
\end{abstract}

Index Terms-Electron energy loss spectroscopy (EELS), metal filled carbon nanotubes (CNTs), microwave plasma-enhanced chemical vapor deposition (MP-CVD), scanning electron microscopy (SEM), transmission electron microscopy (TEM).

\section{INTRODUCTION}

$\mathbf{T}$ HE INTRODUCTION of various materials, such as metals, their compounds, or fullerenes, into carbon nanotubes (CNTs) may lead to a variety of industrial applications. CNTs filled with ferromagnetic metals such as $\mathrm{Fe}, \mathrm{Ni}$, or $\mathrm{Co}$ have significant potential in high-density magnetic storage devices due to their size, anisotropic behavior, and enhanced magnetic coercivities [1]-[6]. In addition, the graphite sheets provide an effective barrier against oxidation, and consequently

Manuscript received January 27, 2006; revised May 1, 2006. This work was supported in part by the Nanotechnology Support Project of the Ministry of Education, Culture, Sports, Science and Technology (MEXT), Japan, carried out at the Research Laboratory of High Voltage Electron Microscopy, Kyushu University, and in part by the Grant-in Aid for Scientific Research (Houga-16651065) of the MEXT. The work at the University of Surrey was supported in part by the EPSRC via a Portfolio Partnership and by the Carbon Based Electronics Program. The review of this paper was arranged by Associate Editor G. Ramanath.

Y. Hayashi and T. Tokunaga are with the Department of Environmental Technology and Urban Planning, Nagoya Institute of Technology, Nagoya 466-8555, Japan.

K. Kaneko is with the Department of Material Science and Engineering, University of Kyushu, Fukuoka 812-8581, Japan.

S. J. Henley, V. Stolojan, J. D. Carey, and S. R. P. Silva are with the Nanoelectronics Centre, School of Electronics and Physical Sciences, Advanced Technology Institute, University of Surrey, Guildford GU2 7XH, U.K.

Digital Object Identifier 10.1109/TNANO.2006.880456 ensure a long-term stability of the ferromagnetic core metal. Moreover, CNTs filled with metals are an effective route to exploit one-dimensional nanocables in various applications [7].

Previously, different groups have tried to synthesize metal-filled CNTs (MF-CNT) and two kinds of methods have been reported so far. One approach has the CNTs filled with molten material through capillary action [8] or with metal oxides using wet chemical techniques after opening the tube top [9]. The other is an in-situ filling method, where metals or metal compounds can be filled into the CNTs by the arc-discharge technique using a graphite anode impregnated with the filling material [10]. Recently, the MF-CNTs by a thermal chemical vapor deposition (CVD) method has also been reported [1]-[6]. Although thermal CVD can produce MF-CNTs in large quantities, it is difficult to produce vertically aligned MF-CNTs. From an application view point, it is desirable to use plasma-enhanced CVD to produce MF-CNTs with highly aligned and well-defined diameters and length. Although we have previously reported Pd-based MF-CNTs using bias-enhanced microwave PECVD (BE-MPECVD) [11], we failed to fill Co metal into the nanotubes using only a Co catalyst layer on the $\mathrm{Si}$ substrate. The Co particle was detached from the substrate and remained at the tip of the growing Co-based CNTs. In order to realize spin electronic applications by using MF-CNTs, it is required to fill the ferromagnetic metals into the tubes of aligned CNTs.

In this paper, we report the Pd/Co-based MF-CNTs synthesized by a BE-MPECVD using a combination of Pd and Co catalyst layers. BE-MPECVD grown MF-CNTs were characterized in detail by scanning electron microscopy (SEM) and transmission electron microscopy (TEM), as well as energy filtered electron energy loss spectroscopy (EELS), and their growth mechanisms were modeled to describe these results. We also carried out visible Raman spectroscopy to compare the microstructure between Pd/Co-based MF-CNTs and Pd-based MF-CNTs or Co-based hollow nanotubes (CNTs).

\section{EXPERIMENTAL}

The MF-CNTs were grown by MPECVD using a $2.45 \mathrm{GHz}$, $1.5 \mathrm{~kW}$ microwave power supply. The substrate temperature was controlled by a radio frequency graphite heater. A negative bias was applied to the substrate holder to grow vertically aligned MF-CNTs on the substrate. The Si substrates with a thin native oxide were cleaned in acetone by ultrasonication followed by deionized water and, finally, dried using a nitrogen 
blower prior to the catalyst deposition. The substrates were then treated in an acid solution $\left(\mathrm{H}_{2} \mathrm{SO}_{4}: \mathrm{H}_{2} \mathrm{O}_{2}=4: 1\right)$ to enhance the thickness of the oxide layer. Thin films of Co $(6 \mathrm{~nm})$ and Pd $(9 \mathrm{~nm})$ were deposited on Si substrates by a thermal evaporation. After deposition of the catalyst layers, the substrates were transferred in air to the CVD chamber. The chamber was pumped down to a base pressure of $10^{-2}$ Torr. The substrates were heated to $750{ }^{\circ} \mathrm{C}$ and held at this temperature for $10 \mathrm{~min}$ to sinter the catalyst layers. The feed gas, methane $\left(\mathrm{CH}_{4}\right)$, was introduced immediately and the concentration of $\mathrm{H}_{2}$ gas was adjusted to achieve a $\mathrm{CH}_{4} / \mathrm{H}_{2}$ ratio of $50 / 50 \mathrm{sccm}$ at a total pressure of 20 Torr. A microwave power of $600 \mathrm{~W}$ was employed and a negative bias was applied to the substrate holder to grow vertically aligned MF-CNTs on the substrate. The MF-CNTs were grown for $10 \mathrm{~min}$ under the conditions where a negative bias of $400 \mathrm{~V}$ was applied.

The MF-CNTs were observed using scanning microscopy (Hitachi S-300, FEI Philips XL30 sFEG) and analyzed using a Philips CM200TEM with a $\mathrm{LaB}_{6}$ filament, fitted with a GIF2000 spectrometer. The Raman spectra were measured in the back-scattering geometry using the $514.5 \mathrm{~nm}$ line of an $\mathrm{Ar}^{+}$ ion laser at room temperature in the spectral range from 900 to $1800 \mathrm{~cm}^{-1}$ with a resolution of $1.0 \mathrm{~cm}^{-1}$.

\section{RESULTS AND DISCUSSION}

Fig. 1(a) shows the SEM images of the Pd/Co-based MF-CNTs grown on Si substrates, and in Fig. 1(b), the evolution of the catalyst after just $3 \mathrm{~min}$ growth of MF-CNTs. The final MF-CNTs are well aligned and uniform in size on the $\mathrm{Si}$ substrate. At lower negative substrate biases below $300 \mathrm{~V}$, randomly oriented sparse CNTs (not MF-CNTs) were observed. This result indicates that a high negative substrate bias above $400 \mathrm{~V}$ is necessary to obtain well-aligned and dense Pd/Co-based MF-CNTs. Fig. 1(a) shows that MF-CNTs are well aligned with uniform diameter and length $(1 \mu \mathrm{m})$ on $\mathrm{Si}$ substrates. It is seen from Fig. 1(b), that the $\mathrm{Pd} /$ Co breaks into small size (about 60-100 $\mathrm{nm}$ diameter) uniformly due to surface tension, as well as the stress due to mismatch of the thermal expansion coefficients during the initial growth stage. For the case of only Co catalyst layers, the particle was found to form spherical islands; different from that observed for the $\mathrm{Pd} / \mathrm{Co}$ catalyst layers. The catalyst for the growth of $\mathrm{Pd} / \mathrm{Co}$-based MF-CNTs should be in a molten state to encapsulate metals inside the tubes. The temperature of growth of $\mathrm{Pd} / \mathrm{Co}$-based MF-CNTs in our case is far lower than the melting point of bulk Pd $\left(1552^{\circ} \mathrm{C}\right)$ and $\mathrm{Co}\left(1495^{\circ} \mathrm{C}\right)$. It is well known that the surface-to-volume ratio with respect to nanosize particle can lower the melting point. However, Pd/Co-based MF-CNTs can grow even from particles with diameters from $60-100 \mathrm{~nm}$.

TEM images revealed both multiwall CNTs (MW-CNTs) and the herringbone (HB) or the stacked cups structure structures on the same substrate, as shown in Fig. 2(a). In both structures, there is the presence of the HB structure. It is reported that the formation of HB-shaped CNTs could be attributed to nitrogen doping, in which nitrogen atoms are generally bonded to carbon atoms in $\mathrm{sp}^{2}$ pyridine-like and $\mathrm{sp}^{3}$ bridgehead-nitrogen types [12]. Residual nitrogen may be a nitrogen precursor for
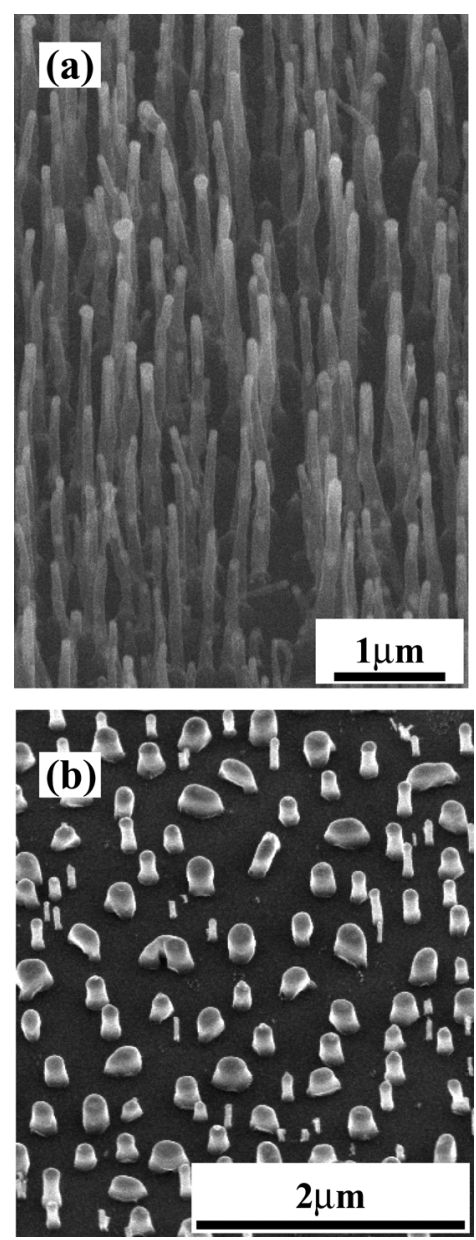

Fig. 1. (a) Scanning electron microscopy images of Pd/Co-based MF-CNTs grown on $\mathrm{Si}$ substrates and (b) the evolution of the catalyst of $\mathrm{Pd} / \mathrm{Co}$ layers after 3 min growth of MF-CNTs.
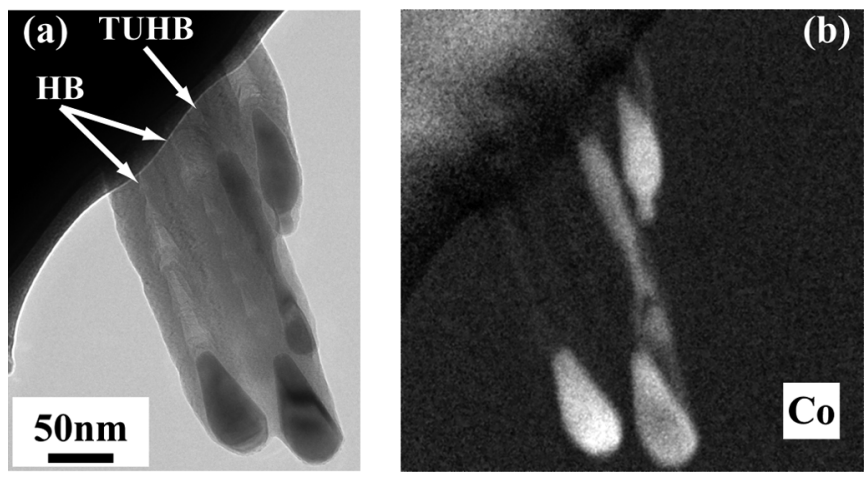

Fig. 2. (a) Transmission electron microscopy image of Pd/Co-based MF-CNTs grown on the Si substrate and (b) Co elemental map inside tubes measured by energy filtered electron energy loss spectroscopy. Metal encapsulation is clearly visible in Fig. 1(b).

the synthesis of HB-shaped CNTs. The exact reason for existing both the tube with $\mathrm{HB}$ and the HB structures in the same substrate is not known up to now. Combining the TEM image [Fig. 2(a)] and the EELS elemental map of Co [Fig. 2(b)], the Co metal was successfully filled inside the MW-CNTs. In the case of HB-shaped structure, Co metal was encapsulated only in the tips. A magnified HRTEM images, as shown in Fig. 3, reveal that the interlayer spacing of a graphite layer is $0.34 \mathrm{~nm}$, consisting with the (002) plane lattice parameter of graphite. 


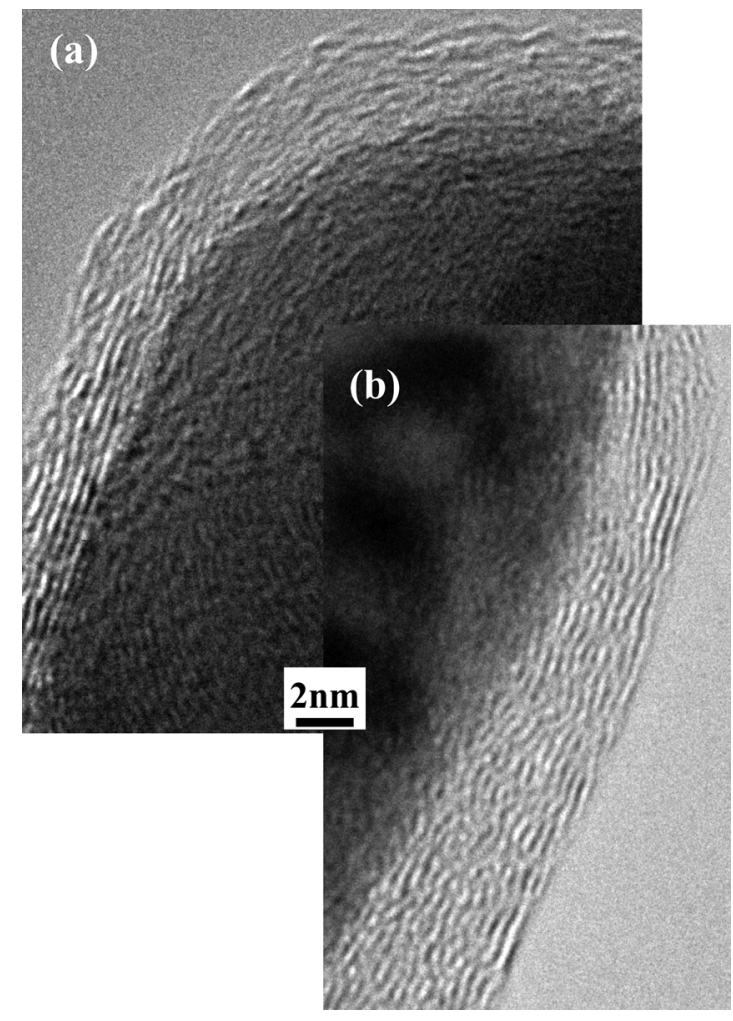

Fig. 3. High-resolution transmission electron microscopy images of $\mathrm{Pd} / \mathrm{Co}-$ based MF-CNTs grown on the Si substrate.

The graphite layers are parallel to the tube axis. Although some amorphous material was observed inside the tube walls for the Pd-based MF-CNTs [11], almost no amorphous material is observed for Pd/Co-based MF-CNTs. Pd metal is generally considered as an inactive metal and Co metal is considered as an active metal in catalyst. Therefore, the reactivity of the catalyst is enhanced by combining Co with $\mathrm{Pd}$ and leads to a reduction of the amorphous nature inside the tube walls for the $\mathrm{Pd} / \mathrm{Co}$-based MF-CNTs. A large number of linear contrast features were observed around the tip for Pd-based MFCNTs due to a lattice strain induced by the solidification of Pd during cooling after growth [11]. In contrast to the Pd-based MF-CNTs, no linear contrast around the tip for Pd/Co-based MF-CNTs. This indicates no lattice strain was induced by the solidification of $\mathrm{Pd} / \mathrm{Co}$ during cooling after growth.

EELS analysis was performed to identify the composition of the material inside the CNTs, as shown in Fig. 4. On the basis of elemental analysis, we have observed not only $\mathrm{Pd}$ and Co but $\mathrm{Si}$ and Mo (not shown in Fig. 4) elements. Si and Mo might be incorporated into the metal within the CNTs from the Si substrate. In the previous study, we have also observed $\mathrm{Si}$ and Mo atoms for the Pd-based MF-CNTs [11]. The elemental maps also clearly reveal that both Pd and Co metals are found inside tubes uniformly. This result indicates that combining Co with $\mathrm{Pd}$ is essential to fill Co metal inside tubes for producing Co filled CNTs.

The first-order Raman spectra of MF-CNTs consists of two broad peaks located at $1590 \mathrm{~cm}^{-1}$ (the $G$ peak) and $1355 \mathrm{~cm}^{-1}$ (the $D$ peak), as shown in Fig. 5. In comparison with $\mathrm{Pd} / \mathrm{Co}$-based MF-CNTs, the Raman spectra of Pd-based MF-CNTs reported previously [11], and multiwall
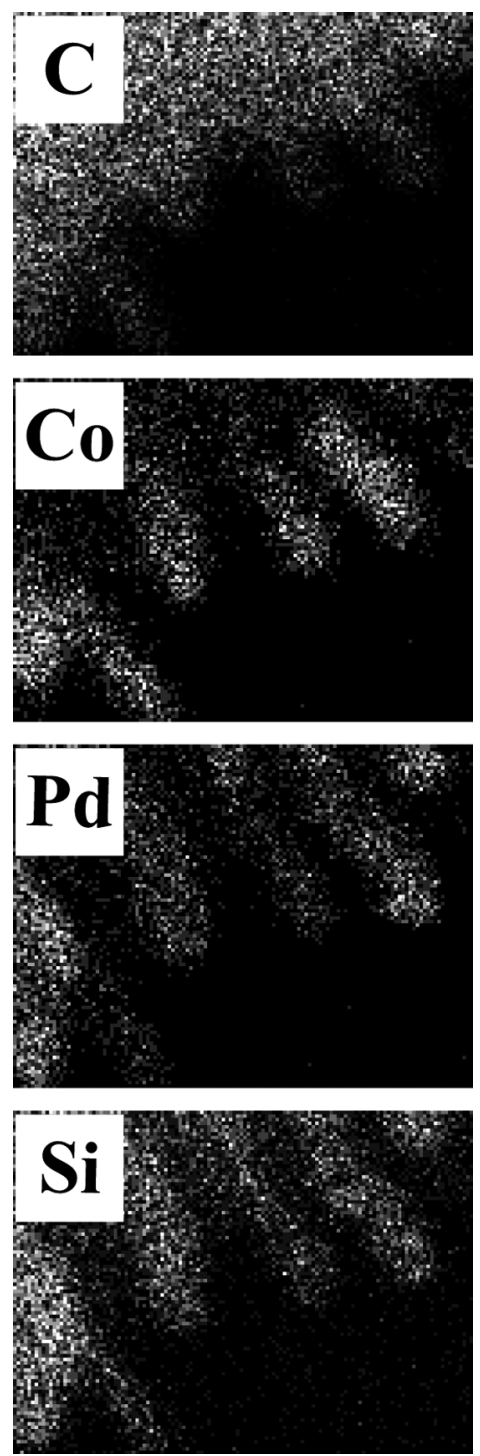

Fig. 4. C, Co, Pd, and Si elemental maps inside tubes measured by energy dispersive X-ray spectroscopy.

CNTs (MWCNTs) grown using Co catalyst particles where the catalyst metal was not filled inside the tubes are shown in Fig. 5(a) and (b), respectively. The $G$ and $D$ peaks are attributed to the in-plain symmetric C-C stretching $\left(E_{2 g}\right)$ and the graphite materials with small crystallite sizes or so-called disordered graphite, respectively. Raman spectra show an additional peak at about $1620 \mathrm{~cm}^{-1}$ (denoted as $D^{\prime}$ ). The origin of the $D$ and $D^{\prime}$ bands in other forms carbon materials has been explained as disorder-induced features, caused by the finite-particle size effects or lattice distortion [13]. Although, the Raman intensity ratio between $D$ and $G$ bands $\left(I_{D} / I_{G}\right)$ of $\mathrm{Pd} / \mathrm{Co}$-based MF-CNTs is always larger than that from the MWCNTs in the experiment, a low intensity of the $D$ band, as well as a low value of the $I_{D} / I_{G}$, indicates high quality of Pd/Co-based MF-CNTs, as shown in Fig. 5(c). While the broadening of $G$-peak was observed for Pd-based MF-CNTs [11], the full-width at $1 / 2$ maximum (FWHM) of the $G$-peak for Pd/Co-based MF-CNTs was almost the same as that of MWCNTs. Furthermore, no shift of the $G$-peak position was observed indicating that the structural change from graphite to 


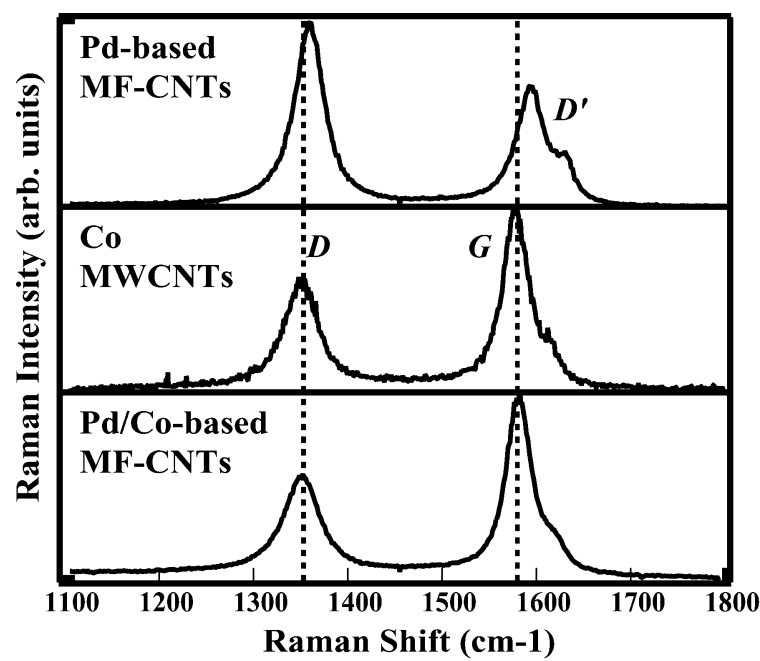

Fig. 5. Raman spectra of Pd-based MF-CNTs, Co-based CNTs multiwall CNTs, and Pd/Co-based MF-CNTs.

(a)

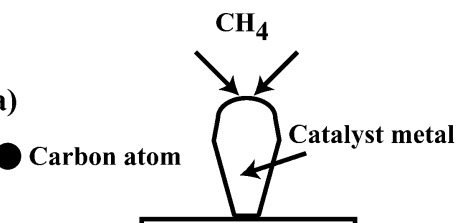

(b1)

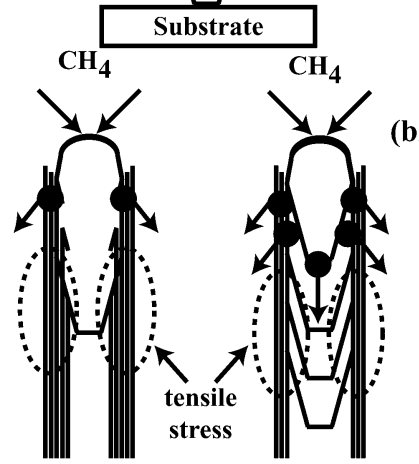

(c1)

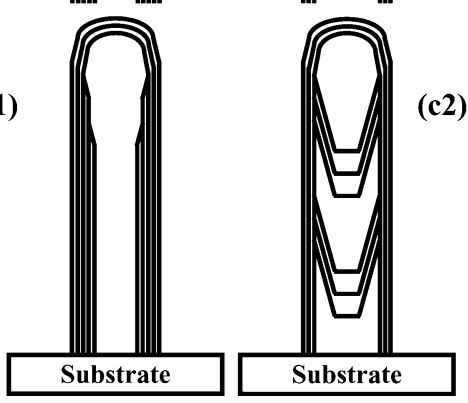

Fig. 6. Summary of the proposed growth model of Pd/Co-based MF-CNTs.

nanocrystalline graphite may not be occurring by filling $\mathrm{Pd} / \mathrm{Co}$ metals inside tubes [14].

Most reports so far, conclude that the catalyst particles are encapsulated on the tip of the MWCNTs. The question arises if there is any reason preventing the metallic catalyst present inside the MF-CNTs. Based on TEM and SEM investigations, we propose the following model to explain the Pd/Co-based MF-CNTs, as schematically shown in Fig. 6. After Pd and Co layer deposition, the mixture of Pd and Co catalyst layers become fragmented into nanoparticles Fig. 6(a). The decomposition of $\mathrm{CH}_{4}$ on the surface of $\mathrm{Pd} / \mathrm{Co}$ nanoparticle results in the formation of bimolecular hydrogen and carbon atoms, and the growth of $\mathrm{Pd} / \mathrm{Co}$-based MF-CNTs is believed to occur via diffusion of carbon atoms through the $\mathrm{Pd} / \mathrm{Co}$ particle leading to the formation of graphite layers at the lower colder zone of the particle. The axis of a MF-CNT growing perpendicular to the substrate coincides with the direction of the applied electrostatic force, resulting in a uniform tensile stress across the entire graphite layer/catalyst particle interface [Fig. 6(b1) and (b2)]. Therefore, carbon uniformly precipitates across the interface and the CNT continues to grow vertically (perpendicular to the substrate) [16]. The key feature of this growth model for the tube with HB is that the precipitation of carbon atom does not occur on the bottom surface of the catalyst particle [Fig. 6(b1) and (b2)]. Therefore, the $\mathrm{Pd} / \mathrm{Co}$ catalyst metals are trapped in the CNT from the basal side when the CNT was filled by Pd and Co metals [Fig. 6(c1)]. On the contrary, the precipitation of carbon atom occurred on the bottom surface of the catalyst particle leads to elevation of the particle to the tip [Fig. 6(c2)]. If the carbon species are supplied in a steady-state manner, the tube will grow vertically for the tube with HB structure, and the HB structure will appear periodically [Fig. 6(c1) and (c2)]. The growth continues until the catalytic activity of the $\mathrm{Pd} / \mathrm{Co}$. In previous reports, metals were considered to be encapsulated in the hollows of CNTs by the capillary force [8], [15]. Although the capillary force was also active in our experiments, encapsulation was thought to start at the beginning of growth of MF-CNTs.

\section{CONCLuSION}

In conclusion, we report the synthesis and characterization of Pd/Co-based MF-CNTs. We have successfully grown well-aligned $\mathrm{Pd} / \mathrm{Co}$-based MF-CNTs with uniform diameter and length $(1 \mu \mathrm{m})$ on Si substrates. TEM revealed multiwall nanotubes and the HB or stacked cups structure on the same substrate. HRTEM revealed that MF-CNTs were composed of highly ordered graphene layers. Based on TEM observation and analysis by EELS showed that Co metal was successfully encapsulated into the CNTs from the basal side by combining Co with Pd metals. Raman results showed a low intensity of the $D$ band, as well as a low value of the $I_{D} / I_{G}$ indicate the high quality of Pd/Co-based MF-CNTs. Moreover, no broadening and no shift of the $G$-peak were observed indicating that the structural change from graphite to nanocrystalline graphite may not be occurred by filling $\mathrm{Pd} / \mathrm{Co}$ metals inside tubes. A growth model for the Pd/Co-based MF-CNTs is proposed based on these experimental results. The key feature of the growth model for the tube with HB structure is that the precipitation of carbon atom does not occur on the bottom surface of the catalyst particle. Finally, owing to the unique properties of the $\mathrm{Pd} /$ Co-based MF-CNTs, they have potential application for tips of scanning probe microscopy or high-density magnetic storage devices. 


\section{REFERENCES}

[1] A. L. Elias, J. A. Rodríguez-Manzo, M. R. McCartney, D. Golberg, A. Zamudio, S. E. Baltazar, F. López-Urías, E. Muñoz-Sandoval, L. Gu, C. C. Tang, D. J. Smith, Y. Bando, H. Terrones, and M. Terrones, "Production and characterization of single-crystal FeCo nanowires inside carbon nanotubes," Nano Lett., vol. 5, pp. 467-472, Jan. 2005.

[2] A. Leonhardt, M. Ritschel, R. Kozhuharova, A. Graff, T. Mühl, R. Huhle, I. Mönch, D. Elefant, and C. M. Schneider, "Synthesis and properties of filled carbon nanotubes," Diamond Relat. Mater., vol. 12, pp. 790-793, Mar.-Jul. 2003.

[3] A. L. Elías, J. A. Rodríguez-Manzo, M. R. McCartney, D. Golberg, A. Zamudio, S. E. Baltazar, F. López-Urías, E. Muñoz-Sandoval, L. Gu, C. C. Tang, D. J. Smith, Y. Bando, H. Terrones, and M. Terrones, "Production and characterization of single-crystal FeCo nanowires inside carbon nanotubes," Nano Lett., vol. 5, pp. 467-472, Jan. 2005.

[4] J. Bao, C. Tie, Z. Xu, Z. Suo, Q. Zhou, and J. Hong, "A facile method for creating an array of metal-filled carbon nanotubes," Adv. Mater., vol. 14, pp. 1483-1486, Oct. 2002.

[5] J. Bao, Q. Zhou, J. Hong, and Z. Xu, "Synthesis and magnetic behavior of an array of nickel-filled carbon nanotubes," Appl. Phys. Lett., vol. 81, pp. 4592-4594, Dec. 2002.

[6] N. Grobert, W. K. Hsu, Y. Q. Zhu, J. P. Hare, H. W. Kroto, D. R. M. Walton, M. Terrones, H. Terrones, P. Redlich, M. Rühle, R. Escudero, and F. Morales, "Enhanced magnetic coercivities in Fe nanowires," Appl. Phys. Lett., vol. 75, pp. 3363-3365, Nov. 1999.

[7] W. Y. Choi, J. W. Kang, and H. J. Hwang, "Structure of ultrathin copper nanowires encapsulated in carbon nanotubes," Phys. Rev. B, Condens. Matter, vol. 68, pp. 193 405-193 436, Nov. 2003.

[8] M. Ajayan and S. Iijima, "Capillarity-induced filling of carbon nanotubes," Nature, vol. 361, pp. 333-334, Jan. 1993.

[9] S. C. Tsang, Y. K. Chen, P. J. F. Harris, and M. L. H. Green, "A simple chemical method of opening and filling carbon nanotubes," Nature, vol. 372, pp. 159-162, Nov. 1994.

[10] C. Guerret-Piécourt, Y. Le Bouar, A. Lolseau, and H. Pascard, "Relation between metal electronic structure and morphology of metal compounds inside carbon nanotubes," Nature, vol. 372, pp. 761-765, Dec. 1994.

[11] Y. Hayashi, T. Tokunaga, Y. Yogata, S. Toh, K. Kaneko, T. Soga, and T. Jimbo, "Synthesis of corn-shape carbon nanofibers on Si and Mo substrates by bias-enhanced microwave plasma chemical vapor deposition," Diamond Relat. Mater., vol. 13, pp. 1198-1202, Apr.-Aug. 2004.

[12] J. W. Jang, C. E. Lee, S. C. Lyu, T. J. Lee, and C. J. Lee, "Structural study of nitrogen-doping effects in bamboo-shaped multiwalled carbon nanotubes," Appl. Phys. Lett., vol. 84, pp. 2877-2879, Apr. 2004.

[13] V. Barbarossa, F. Galluzzi, R. Tomaciello, and A. Zanobi, "Raman spectra of microcrystalline graphite and a-C:H films excited at 1064 nm," Chem. Phys. Lett., vol. 185, pp. 53-55, Oct. 1991.

[14] A. C. Ferrari and J. Robertson, "Interpretation of Raman spectra of disordered and amorphous carbon," Phys. Rev. B, Condens. Matter, vol. 61, pp. 14095-14107, May 2000.

[15] G. Y. Zhang and E. G. Wang, "Cu-filled carbon nanotubes by simultaneous plasma-assisted copper incorporation," Appl. Phys. Lett., vol. 82, pp. 1926-1928, Mar. 2003.

[16] V. I. Merkulov, A. V. Melechko, M. A. Guillorn, D. H. Lowndes, and M. L. Simpson, "Alignment mechanism of carbon nanofibers produced by plasma-enhanced chemical-vapor deposition," Appl. Phys. Lett., vol. 79, pp. 2970-2972, Oct. 2001.

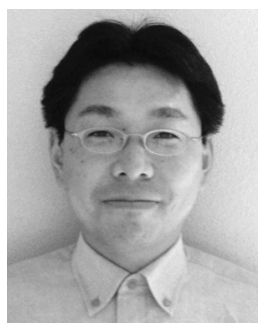

Yasuhiko Hayashi received the B.E., M.E., and D.E. degrees in electrical and computer engineering from the Nagoya Institute of Technology, Nagoya, Japan, in 1990,1992 , and 1999 , respectively.

From 1992 to 1996, he was engaged in research on semiconductor device modeling at Motorola Japan, Ltd., Tokyo, Japan. In 1999, he joined the Department of Environmental Technology and Urban Planning, Nagoya Institute of Technology, as a Research Associate. From June to November 2005, he was with the University of Cambridge, as a Visiting Scientists, during which time he explored the metal filled carbon nanotubes. His research interests are fundamental experiments on carbon nanomaterial synthesis using plasma technology, and its application to spin electronic, as well as nanoelectronic devices.

Dr. Hayashi is a member of the Fullerenes and Nanotubes Research Society and the Japan Society of Applied Physics.

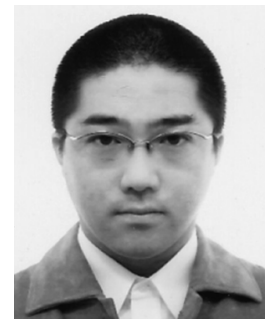

Tomoharu Tokunaga was born in 1981 . He received the B.E. degree in electronics and information engineering and the M.E. degree in environmental technology and urban planning from the Nagoya Institute of Technology, Nagoya, Japan, in 2003 and 2005, respectively. He is currently working towards the Ph.D. degree in the Department of Material Science and Engineering, Kyushu University, Fukuoka, Japan.

His current research topic is fabrication of carbon related material and metal composites by mechanical deformation.

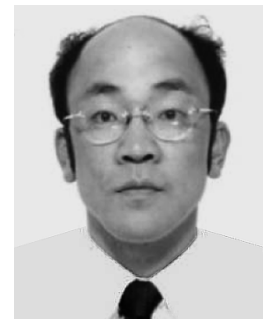

Kenji Kaneko was born in Gumma, Japan, in 1967. $\mathrm{He}$ received the B.Sc. degree in physics from Imperial College, University of London, London, U.K., in 1990 and the Ph.D. degree in physics from Bristol University, Bristol, U.K., in 1995.

After returning to Japan, he was employed as a Postdoctoral Researcher at various institutes, including Tokyo University. After these positions, he joined Kyushu University as an Associate Professor in 2001. His current research interests focus on characterizing various types of materials by transmission electron microscopy, analytical electron microscopy, and three-dimensional electron tomography.

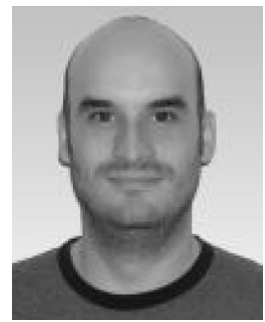

Simon J. Henley received the Ph.D. degree in physics from of the University of Bristol, Bristol, U.K., in 2001.

During this period his main area of research involved study of the effect of defects, such as dislocations, on the luminescence efficiency of GaN-based optoelectronic materials. After this he stayed on at the University of Bristol in a joint chemistry/physics DTI LINK OSDA sponsored collaborative project to develop field emission displays and was involved in fundamental research into pulsed laser ablation and deposition of multicomponent materials such as $\mathrm{ZnO}$ and $\mathrm{LiF}$. Currently, he is a Postdoctoral Research Fellow in the Nano-Electronics Centre in the Multidisciplinary Advanced Technology Institute (ATI), University of Surrey, where he is continuing his research into the application of pulsed laser ablation for the production of nanomaterials. In particular, he is interested in carbon-based material systems for future device applications. Current activities include the deposition of nanostructured carbon materials for sensor applications and the study of quantum sized optical/electrical effects in multilayers/superlattices of amorphous carbon.

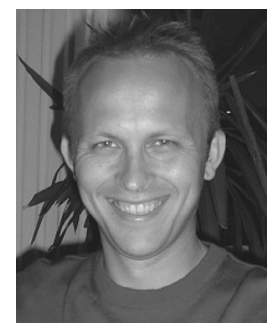

Vlad Stolojan received the B.Sc. in physics from the University of East Anglia, Norwich, U.K., in 1996 and the Ph.D. degree from the University of Cambridge, Cambridge, U.K., in 2001.

In 2001, he was engaged in research in holography-tomography at the Department of Materials Science and Metallurgy, University of Cambridge. In 2001, he joined the School of Engineering, University of Surrey, Surrey, U.K., and since 2004 he has been with the Advanced Technology Institute, University of Surrey, as a Research Fellow. His research interests are electron microscopy (transmission, scanning) and electron energy loss spectroscopy applied to the study of nanomaterials, their morphologies, structures, and electronic properties.

Dr. Stolojan is a member of the Royal Microscopical Society and the Institute of Physics. 


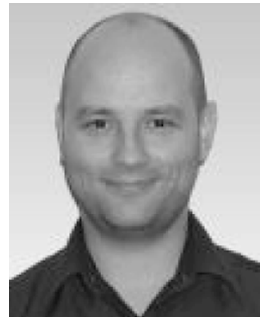

J. David Carey received the B.A. and Ph.D. degrees from the University of Dublin, Trinity College, Dublin, Ireland, in 1992 and 1997, respectively.

In 1998, he joined the Electronic Engineering Department, University of Surrey, Guildford, U.K In 2002, he was appointed a EPSRC Advanced Research Fellow and in 2004 appointed an Assistant Professor in the Advanced Technology Institute, University of Surrey. His research interests include electron transport and electron field emission from carbon-based electronic materials, in particular, from carbon nanotubes. Other interests include defects in materials and electronic devices.

Dr. Carey is a Member of the Institute of Electrical Engineers (U.K.), the American Physical Society, and the Materials Research Society.

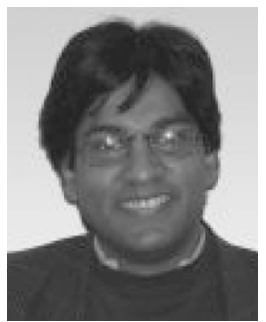

S. R. P. Silva received the B.A. and Ph.D. degrees from the University of Cambridge, Cambridge, U.K., in 1990 and 1994, respectively.

Currently, he is the Director of the Advanced Technology Institute, University of Surrey, Surrey, U.K., and leads the Nano-Electronics Centre. He holds the Chair in Solid State Electronics at the University of Surrey. He has published over 200 papers in refereed journals and made over 250 paper presentations at international conferences. His research interest encompasses a wide range of activities in the areas of nanoelectronics and large-area electronics.

Prof. Silva was awarded the Charles Vernon Boys Medal by the Institute of Physics in 2002, and in 2003, was awarded the Achievement Award by the Institute of Electrical Engineers. In 2003, he was also awarded the Albert Einstein Silver Medal and Javed Husain Prize by UNESCO for contributions to electronic devices. He is a Fellow of the Institute of Physics and the Institute of Electrical Engineers. 